\title{
Non-pegylated liposome-encapsulated doxorubicin citrate plus cyclophosphamide or vinorelbine in metastatic breast cancer not previously treated with chemotherapy: A multicenter phase III study
}

\author{
V. LORUSSO ${ }^{1}$, F. GIOTTA ${ }^{1}$, R. BORDONARO ${ }^{2}$, E. MAIELLO ${ }^{3}$, S. DEL PRETE ${ }^{4}$, V. GEBBIA ${ }^{5}$,

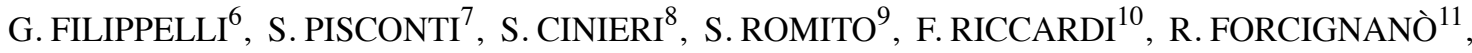 \\ M. CICCARESE ${ }^{11}$, L. PETRUCELLI ${ }^{11}$, V. SARACINO ${ }^{11}$, L.I.LUPO ${ }^{11}$, A. GAMBINO ${ }^{11}$, S. LEO ${ }^{11}$ \\ and G. COLUCCI FOR THE GOIM (GRUPPO ONCOLOGICO DELL'ITALIA MERIDIONALE)
}

\footnotetext{
${ }^{1}$ National Cancer Research Center, Istituto Tumori Giovanni Paolo II, Bari; ${ }^{2}$ Catania Hospital, Catania; ${ }^{3}$ Casa Sollievo della Sofferenza Hospital IRCCS, San Giovanni Rotondo (FG); ${ }^{4}$ Frattamaggiore Hospital, Frattamaggiore (NA); ${ }^{5}$ La Maddalena Hospital, Palermo; ${ }^{6}$ Paola Hospital, Paola (CS); ${ }^{7}$ Taranto Hospital, Taranto; ${ }^{8}$ Brindisi Medical Oncology Department and Breast Unit e Medical Department, European Insitute of Oncology, Milan; ${ }^{9}$ Ospedali Riuniti Hospital, Foggia; ${ }^{10}$ Cardarelli Hospital, Napoli; ${ }^{11}$ Vito Fazzi Hospital, Lecce, Italy
}

Received April 21, 2014; Accepted June 20, 2014

DOI: 10.3892/ijo.2014.2604

\begin{abstract}
We conducted a phase III multicenter randomized trial to compare the efficacy of the combination of liposome encapsulated doxorubicin $\left(\right.$ Myocet $\left.^{\odot}\right)$ plus either cyclophosphamide (MC) or vinorelbine (MV). Since July 2006, 233 patients affected with metastatic breast cancer were randomized to receive the combination of Myocet (M) $60 \mathrm{mg} / \mathrm{m}^{2}$ i.v. plus cyclophosphamide (C) $600 \mathrm{mg} / \mathrm{m}^{2}$ on Day 1 of a 21-day cycle (Arm A) or Myocet (M) at $50 \mathrm{mg} / \mathrm{m}^{2}$ plus vinorelbine (V) $25 \mathrm{mg} / \mathrm{m}^{2}$ i.v. on Day 1 and V $60 \mathrm{mg} / \mathrm{m}^{2}$ orally on Day 8 on a 21-day cycle (Arm B). The primary endpoints of the study was time to progression (TTP); secondary endpoints were RR, toxicity and OS. Response was observed in 53/116 (45.7\%) evaluable patients of Arm A vs. 51/112 (45.5\%) of Arm B, respectively ( $\mathrm{P}=\mathrm{NS}$ ). Median TTP was 41 weeks (95\% CI, 32-51) and 34 weeks (95\% CI, 26-39), for $\mathrm{M} / \mathrm{C}$ and $\mathrm{M} / \mathrm{V}$, respectively ( $\mathrm{P}=0.0234)$. The difference in median OS was not statistically significant (131 vs. 122 weeks; $\mathrm{P}=0.107)$. With regard to toxicity, patients treated with MV showed a slight increase of neutropenia and constipation, as compared to those treated with MC. No clinical signs of cardiotoxicity were observed. The MC combination remains as an unbeaten 'standard' in first line treatment of MBC.
\end{abstract}

Correspondence to: Professor V. Lorusso, National Cancer Research Center, Istituto Tumori Giovanni Paolo II, Bari, Italy

E-mail: vitolorusso@me.com

Key words: liposomal doxorubicin, breast cancer, vinorelbine

\section{Introduction}

Metastatic breast cancer (MBC) is a chronic and incurable disease, with a median survival of approximately 2-3 years. Although advances have been made in the management of MBC, long-term survivors are rare, with 5-year survival rates varying from 5 to $10 \%(1,2)$.

Conventional anthracyclines are among the most widely used agents to treat breast cancer in the adjuvant setting, as well as in metastatic disease (3). In phase II trials the response rate to anthracyclines in patients naïve to chemotherapy is about $50 \%$, however, the clinical use of anthracyclines is limited by dose-related cardiotoxicity, which becomes more relevant with increasing cumulative dose (4-6). The mechanism of anthracycline related cardiotoxicity involves the formation of free radicals that cause lipid perossidation leading to a dose-dependent cardiomyopathy and to congestive heart failure (7-9). Cardiotoxicity can appear during treatment, at the end of therapy, or even years after the end of anthracycline therapy (10). Moreover, doxorubicin associated cardiotoxicity is often unpredictable, because it can happen at lower dose than expected. It was recently reported that this may be related to a genetic predisposition in patients carrying selected polymorphism of the NAD(P)H oxidase and doxorubicin efflux transporters (11).

Non-pegylated liposome-encapsulated doxorubicin citrate, a liposomal formulation of doxorubicin, was designed to reduce cardiotoxicity while preserving antitumor efficacy (12-15). The formulation consists in encapsulation of water-soluble doxorubicin within a phospholipid membrane to prevent doxorubicin from exiting the circulation through the capillary junctions in healthy tissues. Conversely, liposome-encapsulated drug appears to pass easily through the damaged capillaries of 
tumor tissues. Therefore, liposome encapsulated doxorubicin does not easily reach heart tissue and a higher cumulative dose of liposome-encapsulated doxorubicin can be delivered to the patient $(16,17)$. Results from phase III trials demonstrated that liposome-encapsulated doxorubicin and doxorubicin are equivalent in terms of antitumor efficacy (18-20). In particular, Myocet in combination with cyclophosphamide showed an efficacy equivalent to doxorubicin, with a better profile in terms of cardiotoxicity and hematological toxicity (20).

Vinorelbine is a semi-synthetic vinca alkaloid, with leukopenia and constipation as the dose limiting toxicities. Phase II studies have shown a response rate ranging from 35 to $50 \%$ for vinorelbine as first line chemotherapy for metastatic breast cancer (21-23).

This multicenter randomized phase III trial was designed to compare the efficacy and tolerability of the regimen MV vs. MC. Secondary end-points were ORR, safety and OS.

The principal aim of the study was to assess a difference in time to progression (TTP) between M/C (Arm A) and M/V (Arm B). With the use of a one-sided log-rank test with a type I error of 0.1 , we determined that 133 events (disease progression or death from any cause) would be required for an $80 \%$ power to detect an expected minimum difference of 2.5 months in PFS between the two arms in favor of vinorelbine containing arm. With a 1:1 randomization of assignment to study groups and considering a total duration of the study of 60 months (48 months of accrual and 12 months of follow-up), we estimated that we would need to enroll 220 patients to observe 133 events.

\section{Patients and methods}

All eligible patients had histological confirmed locally advanced (LABC) or metastatic breast cancer (MBC) Her-2 negative not previously treated with chemotherapy for advanced disease. Previous treatment with an anthracyclinesbased chemotherapy regimen in the adjuvant or neo-adjuvant setting was allowed (doxorubicin cumulative dose $<240 \mathrm{mg} / \mathrm{m}^{2}$, epirubicin cumulative dose $<360 \mathrm{mg} / \mathrm{m}^{2}$ ). Other inclusion criteria were: age $18-70$ years; PS $(\mathrm{ECOG}) \leq 2$; at least one measurable lesion according to RECIST criteria; previous endocrine therapy allowed; adequate bone marrow, liver and renal function. Main exclusion criteria were: previous chemotherapy for metastatic disease, pregnancy or breast-feeding, serious concomitant systemic disorders, pre-existing sensorial or motor neuropathy, symptomatic brain metastasis, second primary malignancy (except in situ carcinoma of the cervix or adequately treated non-melanoma carcinoma of the skin or other malignancies treated at least 5 years previously with no evidence of recurrence). Randomization method 1:1 was performed and any eligible patient was randomized to receive:

Arm A, non-pegylated liposome encapsulated doxorubicin citrate (M) $60 \mathrm{mg} / \mathrm{m}^{2}$, Day $1 \mathrm{q} 3 \mathrm{w}+$ cyclophosphamide (C) $600 \mathrm{mg} / \mathrm{m}^{2}$, Day $1 \mathrm{q} 3 \mathrm{w}$; Arm B, non-pegylated liposome encapsulated doxorubicin citrate (M) $50 \mathrm{mg} / \mathrm{m}^{2}$, Day $1 \mathrm{q} 3 \mathrm{w}+$ vinorelbine (V) $25 \mathrm{mg} / \mathrm{m}^{2} \mathrm{iv}$, Day $1 \mathrm{q} 3 \mathrm{w}$ and $60 \mathrm{mg} / \mathrm{m}^{2}$ orally, Day $8 \mathrm{q} 3 \mathrm{w}$. Treatment was performed until 6 cycles in case of SD or 8-10 cycles if PR or CR was reached.

Time to progression (TTP) was determined from the date of randomization to the date of assessment of disease progression or death from any cause. TTP curves were calculated by
Table I. Characteristics of the patients.

Arm A

Arm B

Characteristics

no. of patients $(\%)$ no. of patients $(\%)$

Assessable/entered

$116 / 117$

$112 / 116$

Median age (range)

$59(37-71)$

$58(25-70)$

\section{ECOG PS}

0-1

96 (77)

89 (76)

2

$21(23)$

Receptor status

Positive

96 (77)

$93(74)$

Negative

22 (17)

$26(20)$

Unknown

9 (6)

Her-2 status

Positive

8 (6)

8 (6)

Negative

$101(80)$

$105(84)$

Unknown

18 (14)

Prior surgery

$92(74)$

$104(83)$

Prior adjuvant

46 (37)

radiotherapy

Prior hormonal therapy

Prior adjuvant therapy

$98(84)$

103 (89)

with anthracyclines

Dominant site

of disease

Soft tissue

25 (19)

12 (10)

$90(71)$

17 (13)

Viscera

92 (74)

No. of metastatic sites$$
1
$$

$\geq 3$

40 (31)

Kaplan-Meier method. Toxicity was graduated according to NCI-CTC v. 4.0. Objective response, according to RECIST criteria, was evaluated by a tumor assessment performed every 3 cycles. Survival time was measured from the date of randomization to the date of death or lost to follow-up. Overall survival (OS) curves were calculated by Kaplan-Meier method. Cardiotoxicity was defined as appearance of signs and/or symptoms of CHF and/or a decrease in LVEF below normal limit $(<50 \%)$ or a decline $\geq 15 \%$ from baseline value. To evaluate Left Ventricular Ejection Fraction (LVEF) an echocardiography was done at baseline and every 3 cycles in all patients entered into the study.

\section{Results}

From July 2006 through December 2011,233 patients with metastatic breast cancer, and no prior chemotherapy for advanced 
Table II. Response rate in per-protocol evaluable patients according to dominant site of disease.

\begin{tabular}{|c|c|c|c|c|c|}
\hline Dominant site of disease & No. of patients & $\mathrm{CR}$ & PR & SD & $\mathrm{PD}$ \\
\hline \multicolumn{6}{|l|}{ Arm A } \\
\hline Viscera & 81 & 3 & 31 & 28 & 19 \\
\hline Bone & 11 & 0 & 4 & 4 & 3 \\
\hline Soft tissue & 24 & 5 & 10 & 8 & 1 \\
\hline Total $(\%)$ & 116 & $8(7)$ & $45(39)$ & $40(34)$ & $23(20)$ \\
\hline \multicolumn{6}{|l|}{ Arm B } \\
\hline Viscera & 82 & 2 & 37 & 23 & 20 \\
\hline Bone & 15 & 0 & 5 & 8 & 2 \\
\hline Soft tissue & 15 & 1 & 6 & 6 & 2 \\
\hline Total (\%) & 112 & $3(3)$ & $48(43)$ & $37(33)$ & $24(21)$ \\
\hline
\end{tabular}

disease, were enrolled at 18 Italian centers: 117 patients were randomized to receive $\mathrm{MC}$ and 116 patients to receive $\mathrm{MV}$. The results are reported on the basis of data collected on all 233 patients with follow-up through December 2012. The two treatment groups were equally distributed for baseline characteristics with respect to age, disease-free interval, ECOG performance status, estrogen/progesterone receptor status and visceral involvement (Table I). Prior therapies were well matched between the two groups: $84 \%$ in group A vs. $89 \%$ of group B had received anthracyclines as adjuvant therapy, respectively. Up to two lines of previous hormonal treatment were allowed. A median of 6 cycles (range 2-10) of therapy was performed in both arms. With regard to response rate, 116/117 patients in Arm A and 112/116 in Arm B were evaluable per protocol, however, responses were also assessed on intention to treat basis. In fact, 1 patient and 4 patients for Arms A and $\mathrm{B}$, respectively, were not evaluable for early drop out (1 refused to continue, 3 lost to follow-up, 1 drug related death after the first cycle in Arm B). Overall response rate (Table II), per protocol, was 53/116 [45.7\% (95\% CI, 36.5-54.7\%)] for Arm A and 51/112 [45.5\% (95\% CI, 36.3-54.7\%)] for Arm B, respectively $(\mathrm{P}=\mathrm{NS})$. On intent to treat basis, responses were 53/117 [45.29\% (95\% CI, 36.6-54.3\%)] and 51/116 [44\% (95\% CI, 35.3-53.0\%)] for Arms A and B, respectively. The response rate by prognostic factors showed a high degree of comparability between the two treatment groups (Table III). The duration of response was longer for MC treated patients as compared to MV (26 vs. 19 weeks; $\mathrm{P}=0.327$ ). Median TTP (Fig. 1) was 41 (95\% CI, 32-51) and 34 (95\% CI, 26-39) weeks, respectively $(\mathrm{P}=0.0234)$. With a median follow-up of 36 months, there was little difference in the overall survival curves. The median survival was 131 weeks in the MC patients vs. 122 weeks in the MV treated patients $(\mathrm{P}=0.107)$ (Fig. 2).

Safety. Therapy was generally well tolerated in both groups (Table IV). Myelosuppression was the most frequent and severe adverse side effect on both arms, and was the most common cause of dose reduction and delay. Still, dose reductions and delays were not common: median duration of cycles was 21 days on both arms, and patients achieved $90 \%$ of the planned dose intensity. Grade 3 or 4 thrombocytopenia was observed in $2 \%$ of the MC vs. $3 \%$ of MV patients. Grade 3-4 neutro-
Table III. Results with regard to prognostic factors.

\begin{tabular}{|c|c|c|}
\hline & \multicolumn{2}{|c|}{ Percentage of responding patients } \\
\hline & $\mathrm{MC}(\mathrm{N}=116)$ & $\mathrm{MV}(\mathrm{N}=112)$ \\
\hline \multicolumn{3}{|l|}{ Age (years) } \\
\hline$<50$ & 52 & 54 \\
\hline $50-59$ & 45 & 43 \\
\hline$>60$ & 35 & 32 \\
\hline \multicolumn{3}{|l|}{ Disease-free interval } \\
\hline$<2$ years & 44 & 45 \\
\hline$>2$ years & 42 & 43 \\
\hline \multicolumn{3}{|l|}{ ECOG PS } \\
\hline 0 & 48 & 47 \\
\hline 1 & 47 & 45 \\
\hline 2 & 25 & 24 \\
\hline \multicolumn{3}{|l|}{ Receptor status } \\
\hline Positive & 44 & 43 \\
\hline Negative/unknown & 46 & 46 \\
\hline Prior radiotherapy & 43 & 42 \\
\hline Prior hormonal therapy & 44 & 45 \\
\hline $\begin{array}{l}\text { Prior adjuvant therapy } \\
\text { with anthracyclines }\end{array}$ & 43 & 40 \\
\hline \multicolumn{3}{|l|}{ Dominant site of disease } \\
\hline Soft tissue & 75 & 80 \\
\hline Bone & 35 & 38 \\
\hline Viscera & 45 & 44 \\
\hline \multicolumn{3}{|l|}{ No. of metastatic sites } \\
\hline 1 & 45 & 45 \\
\hline 2 & 45 & 45 \\
\hline$\geq 3$ & 38 & 40 \\
\hline
\end{tabular}

penia, however, was significantly lower in the $\mathrm{MC}$ arm: $16 \%$ of MC patients as compared to $32 \%$ of MV patients $(\mathrm{P}=0.008)$. 


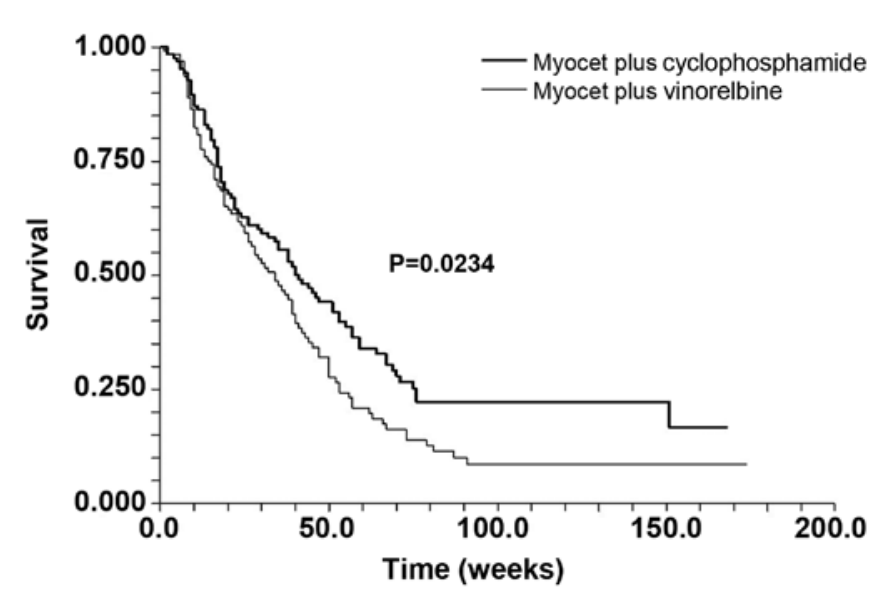

Figure 1. Time to progression (TTP) of patients treated with Myocet plus cyclophosphamide vs. those treated with Myocet plus vinorelbine.

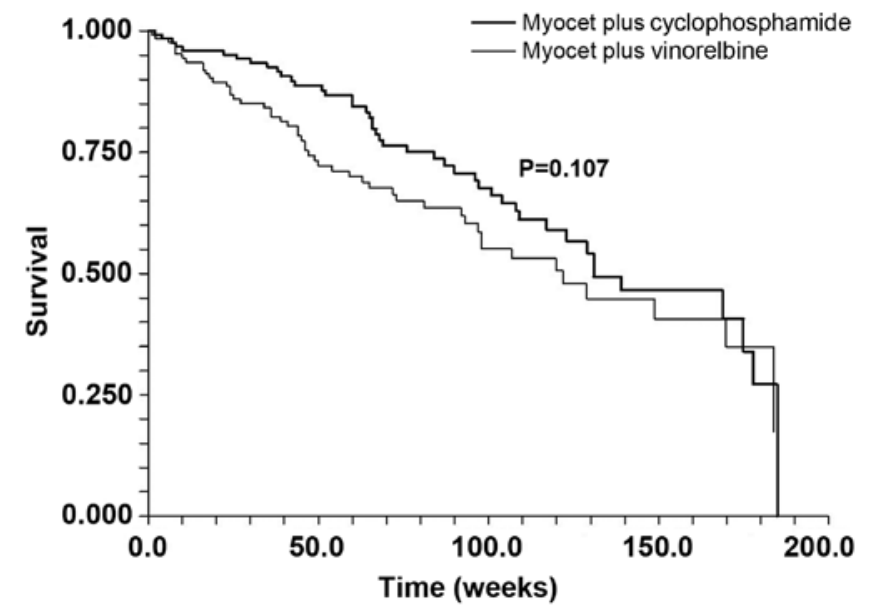

Figure 2. Overall survival of patients treated with Myocet plus cyclophosphamide vs. those treated with Myocet plus vinorelbine.

Table IV. Toxicity.

\begin{tabular}{|c|c|c|c|c|}
\hline \multirow[b]{2}{*}{ Toxicity } & \multicolumn{2}{|c|}{$\operatorname{Arm} A(N=117)$} & \multicolumn{2}{|c|}{ Arm B $(\mathrm{N}=116)$} \\
\hline & Grade I-II (\%) & Grade III-IV (\%) & Grade I-II (\%) & Grade III-IV (\%) \\
\hline Leucopenia & $25(21)$ & $19(16)$ & $23(20)$ & $37(32)$ \\
\hline Febrile neutropenia & - & $8(7)$ & - & $18(16)$ \\
\hline Anemia & $40(34)$ & 3 (3) & $39(34)$ & $7(6)$ \\
\hline Thrombocytopenia & $10(9)$ & $2(2)$ & $9 \quad(8)$ & 4 (3) \\
\hline Nausea/vomiting & $49(42)$ & $2(2)$ & $30(26)$ & $3(3)$ \\
\hline Mucositis & $20(17)$ & $3 \quad(3)$ & $19(16)$ & $9(8)$ \\
\hline Diarrhoea & 4 (3) & 1 (1) & $13(11)$ & 1 (1) \\
\hline Asthenia & $17(15)$ & $2(2)$ & $15(13)$ & $1 \quad(1)$ \\
\hline Constipation & $3(3)$ & 1 (1) & 10 & 8 (7) \\
\hline Hair loss & $43(37)$ & $73(62)$ & $42(36)$ & $70(60)$ \\
\hline Neuropathy & $3(3)$ & - & $4 \quad(3)$ & $1 \quad(1)$ \\
\hline Cardiac toxicity & 8 (7) & - & $9(8)$ & - \\
\hline
\end{tabular}

G-CSF was administered in $35 \%$ of MV cycles vs. $19 \%$ of MC cycles. Eight patients (7\%) on the MC arm compared with 18 patients $(16 \%)$ on the MV arm developed neutropenic fever requiring IV antibiotics and/or hospitalization. Additionally, fewer RBC transfusions were required with MC (5 transfusions to 3 patients) relative to MV (10 transfusions to 7 patients). The incidence of all grade mucositis/stomatitis was comparable in both arms although a slight increase in percent of grade 3-4 mucositis was observed in MV arm (8 vs. 3\%). With regard to gastrointestinal toxicity, incidence of grade 3-4 diarrhea was negligible in both arms (1\%). However, grade 1-2 diarrhea was more frequent on MV as compared to MC (11 vs. 3\%; $\mathrm{P}=0.042$ ). Conversely, constipation was significantly higher on MV arm (16 vs. $4 \%$; $\mathrm{P}=0.003$ ), with $7 \%$ of grade $3-4$.

With regard to cardiotoxicity, overall 17/233 (7\%) patients after 6-8 cycles of either arm, developed a decrease of LVEF below $50 \%$, or showed $>15 \%$ decline of LVEF below basal examination, without any clinical sign of or symptom. Most of these patients $(75 \%)$ were previously treated with anthracyclines. All patients recovered after appropriate treatment. The median dose of non-pegylated liposomal doxorubicin administered was $420 \mathrm{mg}$ (range 120-660). No patient developed palmar-plantar erythrodisesthesia which is a common side effect with pegylated liposomal doxorubicin. Moreover, we observed only one treatment related death in Arm B, because of sepsis.

\section{Discussion}

Doxorubicin is a mainstay in the treatment of breast cancer. In fact, this drug is generally included in the treatment of early as well as of advanced/metastatic disease. However, the most important drawback in its use is the development of cardiotoxicity, which is fatal in more than $5 \%$ of the patients when cumulative dose of $450 \mathrm{mg}^{2}$ is reached (4-6). This risk is particularly high when patients with advanced disease have 
been already treated with anthracyclines as a component of their adjuvant treatment of early disease. Thus, doxorubicin cardiac toxicity can limit patient ability to receive further potentially active therapy, in particular when the tumor is responding to the current treatment including anthracyclines.

With this regard, considerable research has been undertaken to reduce the potential cardiotoxicity of this drug. Drugs having potential protective effect as dexrazoxane $(24,25)$ or the administration of doxorubicin as continuous infusion (26), have been proposed as tools able to reduce risk of hearth damage, but none has been considered really effective. Non-pegylated liposomal doxorubicin $\left(\right.$ Myocet $\left.^{\circ}\right)$ is a unique formulation of doxorubicin, able to alter the tissue distribution of this doxorubicin. Pharmacokinetic studies have demonstrated that doxorubicin in Myocet is bioavailable, metabolized and excreted in a manner similar to that of conventional doxorubicin, but in a slower rate sparing myocardial cells. Moreover, a number of clinical studies (18-20) have demonstrated comparable activity and lesser toxicity of Myocet vs. conventional doxorubicin. In addition, data from a phase III randomized controlled clinical trial, comparing Myocet plus cyclophosphamide (MC) vs. adriamycin plus cyclophosphamide $(\mathrm{AC})$ in advanced breast cancer, demonstrated that Myocet significantly reduced the cumulative cardiac toxicity, while providing comparable antitumor efficacy. In fact, in this study which enrolled 296 patients, an objective response of $43 \%$ was observed in both arms, with a significant reduction of cardiotoxicity (6 vs. $21 \% ; \mathrm{P}=0.0002)$ in favor of $\mathrm{MC}$ patients (20). A further phase III study (19) randomized 160 patients to receive cyclophosphamide $600 \mathrm{mg} / \mathrm{m}^{2}$ plus either epirubicin $75 \mathrm{mg} / \mathrm{m}^{2}$ or liposomal doxorubicin $75 \mathrm{mg} / \mathrm{m}^{2}$. No significant differences were observed in the rate of asymptomatic reduction in LVEF (11 vs. 10\%). In this study, no patient developed clinical heart failure. In 2010, the Cochrane Library reported a systematic review of the different anthracycline compounds and their cardiotoxicity (27). Studies by Harris et al (18) and Batist et al (20) were analyzed together, and authors concluded that nonpegylated liposomal anthracyclines did reduce the overall risk of cardiotoxicity $(\mathrm{RR}=0.38, \mathrm{P}<0.0001)$ and the risk of clinical heart failure $(\mathrm{RR}=0.20, \mathrm{P}=0.02)$.

The aim of the present study was to compare the efficacy of the regimen Myocet plus vinorelbine (MV) vs. the standard $\mathrm{MC}$ in a population mostly pretreated with anthracyclines as adjuvant treatment. Secondary end-points of the study were ORR, safety and OS.

The rationale of the choice of the association of Myocet and vinorelbine as comparator of the standard Myocetcyclophosphamide was the synergism observed in breast cancer cell lines between doxorubicin and vinorelbine (28). In cell lines, an increased p38 activity was demonstrated following vinorelbine, with unchanged mitogen-activated protein kinase (MAPK) activity and p53 expression. On the other hand, doxorubicin treatment resulted in increased p53 expression, without changes in MAPK or p38 activity. This different mechanism of action may result in increased cell apoptosis in vitro as well as in vivo. Nevertheless, the first randomized trial of the combination of doxorubicin plus vinorelbine vs. doxorubicin alone, reported disappointing results (29) with no difference in response rate as well as in overall survival between doxorubicin alone and the combination with vinorelbine. However, following this study, Pawlicki et al (30) reported an objective response rate of nearly $80 \%$, in 38 chemotherapy naïve metastatic breast cancer treated with doxorubicin plus vinorelbine, and Lorvidhaya et al showed an overall response of $67 \%$ in 27 patients (31) with this combination. In addition, few years later, a Scandinavian group reported the results of a phase III study of 387 patients who were randomly assigned to receive IV epirubicin $90 \mathrm{mg} / \mathrm{m}^{2}$ on Day 1 plus vinorelbine $25 \mathrm{mg} / \mathrm{m}^{2}$ on Days 1 and 8 or epirubicin alone at dosage of $90 \mathrm{mg} / \mathrm{m}^{2} \mathrm{IV}$ on Day 1 (32). Both regimens were given every 3 weeks for a maximum of 1 year but discontinued prematurely in the event of progressive disease or severe toxicity. In addition, epirubicin was discontinued at a cumulative dose of $1000 \mathrm{mg} / \mathrm{m}^{2}$ (950 $\mathrm{mg} / \mathrm{m}^{2}$ from June 1999). It is noteworthy that prior anthracycline-based adjuvant chemotherapy and prior chemotherapy for metastatic breast cancer was not allowed. Overall response rates to vinorelbine and epirubicin, and epirubicin alone, were 50 and $42 \%$, respectively $(\mathrm{P}=0.15)$. The complete response rate was significantly superior in the combination arm (17 vs. $10 \%$; $\mathrm{P}=0.048)$ as was median duration of progression-free survival (10.1 vs. 8.2 months; $\mathrm{P}=0.019)$. Median survival was similar in the two arms (19.1 vs. 18.0 months; $\mathrm{P}=0.50)$. Leukopenia related complications, stomatitis and peripheral neuropathy were more common in the combination arm. The incidences of cardiotoxicity and constipation were similar in both arms. The Authors concluded that addition of vinorelbine to epirubicin conferred a significant advantage in terms of complete response rate and progression-free survival, but not in terms of survival.

The results of the present study demonstrate that the combination of Myocet plus cyclophosphamide had a significantly longer TTP compared to Myocet plus vinorelbine while the two combinations showed similar response rate and overall survival. Also safety of the two combination was comparable, except for a higher incidence of leukopenia and neurotoxicity in MV arm.

In conclusion, the combination of Myocet plus cyclophosphamide showed a good profile of efficacy and safety in a population with previous exposure to anthracyclines in the early phase of the disease and remains an unbeaten standard in the treatment of advanced/metastatic breast cancer.

\section{References}

1. Clark GM, Sledge GW Jr, Osborne CK and McGuire WL: Survival from first recurrence: relative importance of prognostic factors in 1,015 breast cancer patients. J Clin Oncol 5: 55-61, 1987.

2. Greenberg PA, Hortobagyi GN, Smith TL, Ziegler LD, Frye DK and Budzar AU: Long-term follow-up of patients with complete remission following combination chemotherapy for metastatic breast cancer. J Clin Oncol 14: 2197-2205, 1996.

3. Launchbury AP and Habbubi N: Epirubicin and doxorubicin: a comparison of their characteristics, therapeutic activity and toxicity. Cancer Treat Rev 3: 197-228, 1993.

4. Smith LA, Cornelius VR, Plummer CJ, Levitt G, Verrill M, Canney $\mathrm{P}$ and Jones A: Cardio-toxicity of anthracycline agents for the treatment of cancer: systematic review and meta-analysis of randomized controlled trials. BMC Cancer 10: 337, 2010.

5. Von Hoff DD, Layard MW, Basa P, et al: Risk factors for doxorubicin-induced congestive heart failure. Ann Intern Med 91: 710-717, 1979.

6. Bristow MR (ed): Anthracycline cardio-toxicity. In: DrugInduced Heart Disease. Elsevier, New York, pp191-215, 1980.

7. Jackson JA, Reeves JP, Muntz KH, et al: Evaluation of free radical effects and catecholamine alterations in adriamycin cardio-toxicity. Am J Pathol 117: 140-153, 1984. 
8. Rajagopalan S, Politi PM, Sinha BK, et al: Adriamycin induced free radical formation in the perfused rat heart: implications for cardio-toxicity. Cancer Res 48: 4766-4769, 1988.

9. Myers CE, McGuire WP, Liss RH, et al: Adriamycin: the role of lipid peroxidation in cardiac toxicity and tumor response. Science 197: 165-167, 1977.

10. Steinherz LJ, Steinherz PG, Tan CT, Heller G and Murphy ML: Cardiac toxicity 4 to 20 years after completing anthracycline therapy. JAMA 266: 1672-1677, 1991

11. Wojnowski L, Kulle B, Schirmer M, et al: NAD(P)H oxidase and multidrug resistance protein genetic polymorphisms are associated with doxorubicin induced cardio-toxicity. Circulation 112: 3754-3762, 2005.

12. Tardi PG, Boman NL and Cullis PR: Liposomal doxorubicin. J Drug Target 4: 129-140, 1996.

13. Kanter PM, Bullard GA, Ginsberg RA, et al: Comparison of the cardiotoxic effects of liposomal doxorubicin (TLC D-99) versus free doxorubicin in beagle dogs. In Vivo 7: 17-26, 1993.

14. Kanter PM, Klaich G, Bullard GA, et al: Preclinical toxicology study of liposome encapsulated doxorubicin (TLC D-99) given intraperitoneally to dogs. In Vivo 8: 975-982, 1994.

15. Bally MB, Nayar R, Masin D, Hope MJ, Cullis PR and Mayer LD: Liposomes with entrapped doxorubicin exhibit extended blood residence times. Biochim Biophys Acta 1023 133-139, 1990

16. Wang X,Yang L, Chen Z and Shin DM: Application of nanotechnology in cancer therapy and imaging. CA Cancer J Clin 58: 97-110, 2008

17. Symon Z, Peyser A, Tzemach D, et al: Selective delivery of doxorubicin to patients with breast carcinoma metastases by stealth liposomes. Cancer 86: 72-78, 1999.

18. Harris L, Batist G, Belt R, et al: Liposome-encapsulated doxorubicin compared with conventional doxorubicin in a randomized multicenter trial as first-line therapy of metastatic breast carcinoma. Cancer 94: 25-36, 2002.

19. Chan S, Davidson N, Juozaityte E, et al: Phase III trial of liposomal doxorubicin and cyclophosphamide compared with epirubicin and cyclophosphamide as first-line therapy for metastatic breast cancer. Ann Oncol 15: 1527-1534, 2004.

20. Batist G, Ramakrishnan G, Rao CS, et al: Reduced cardiotoxicity and preserved antitumor efficacy of liposome-encapsulated doxorubicin and cyclophosphamide compared with conventional doxorubicin and cyclophosphamide in a randomized, multicenter trial of metastatic breast cancer. J Clin Oncol 19: 1444-1454, 2001.
21. Romero A, Rabinovich MG, Vallejo CT, et al: Vinorelbine as first line chemotherapy for metastatic breast carcinoma. J Clin Oncol 12: 336-341, 1994.

22. Smith IE: Navelbine in combination chemotherapy for advanced breast cancer. J Cancer Res Clin Oncol 116: 1052, 1990.

23. Abeloff MD: Vinorelbine (Navelbine) in the treatment of breast cancer: a summary. Semin Oncol 22: 1-4, 1995.

24. Swain SM, Whaley FS, Gerber MC, et al: Cardioprotection with dexrazoxane for doxorubicin containing therapy in advanced breast cancer. J Clin Oncol 15: 1318-1332, 1997.

25. Swain SM, Whaley FS, Gerber MC, et al: Delayed administration of dexrazoxane provides cardioprotection for patients with advanced breast cancer treated with doxorubicin-containing therapy. J Clin Oncol 15: 1333-1340, 1997.

26. Legha SS, Benjamin RS, Mackay B, et al: Reduction of doxorubicin cardiotoxicity by prolonged continuous intravenous infusion. Ann Intern Med 19: 133-139, 1982.

27. Van Dalen EC, Michiels EM, Caron HN and Kremer LC: Different anthracycline derivatives for reducing cardiotoxicity in cancer patients. Cochrane Database Syst Rev 5: CD005006, 2010.

28. Liemm AA, Appleyard MV, O'Neill MA, Hupp TR, Chamberlain MP and Thompson AM: Doxorubicin and vinorelbine act independently via p53 expression and p38 activation respectively in breast cancer cell lines. Br J Cancer 88: 1281-1284, 2003.

29. Norris BI, Pritchard KI, James K, et al: Phase III comparative study of vinorelbine combined with doxorubicin versus doxorubicin alone in disseminated metastatic/recurrent breast cancer. National Cancer Institute of Canada Clinical Trials Group Study MA8. J Clin Oncol 18: 2385-2394, 2000.

30. Pawlicki MI, Rolski J, Zaluski J, Siedlecki P, Ramlau C and Tomzak P: A phase II study of intravenous navelbine and doxorubicin combination in previously untreated advanced breast carcinoma. Oncologist 7: 205-209, 2002.

31. Lorvidhaya VI, Kamnerdsupaphon P, Chitapanarux I, et al: Combination of vinorelbine + doxorubicin in advanced breast cancer. Gan To Kagaku Ryoho 30: 1131-1138, 2003.

32. Ejlertsen B1, Mouridsen HT, Langkjer ST, Andersen J, Sjöström J and Kjaer M; Scandinavian Breast Group Trial (SBG9403): Phase III study of intravenous vinorelbine in combination with epirubicin versus epirubicin alone in patients with advanced breast cancer: a Scandinavian Breast Group Trial (SBG9403). J Clin Oncol 22: 2313-2320, 2004. 